\title{
Propuesta de un PRograma de CAPACITACIÓN CIENTÍFICA (PCC) PARA LA ADQUISICIÓN DE COMPETENCIAS INVESTIGADORAS EN ENFERMERÍA
}

Proposal of a scientific training Program (STP) FOR THE ACQUISITION OF RESEARCH COMPETENCIES IN NURSING ONE OF THE BARRIERS IN NURSING RESEARCH IS NURSES FEELING OF LACK IN RESEARCH TRAINING

Azucena Santillán-García

Hospital Universitario de Burgos

Santillan-Garcia, A. (2020). Propuesta de un programa de capacitación científica (PCC) para la adquisión de competencias investigadoras en enfermería. Revista Ene De EnfermeríA, 14(3). Consultado de http://ene-enfermeria.org/ojs/ index.php/ENE/article/view/1110 


\section{Resumen}

Una de las barreras para la investigación en cuidados es la necesidad sentida de las enfermeras de formación en investigación. El objetivo de esta publicación es mostrar la estructura y contenidos de un curso de formación continuada en investigación denominado "Programa de capacitación científica" (PCC) con el que se pretende disminuir la mencionada barrera. EI PCC consta de 3 módulos con sus correspondientes contenidos teóricos y prácticos, y su sistema de evaluación.

\section{Palabras Clave}

Educación en enfermería, educación basada en competencias, investigación

\section{Abstract}

Proposal of a scientific training program (STP) for the acquisition of research competencies in nursing One of the barriers in nursing research is nurses feeling of lack in research training. The aim of this paper is to show the structure and contents of a training course called "Scientific Training Program" (STP) with which it is intended to reduce the aforementioned barrier. STP has three modules with their corresponding theoretical and practical contents and evaluation system.

\section{Key Words}

Nursing education, competency based education, research 


\section{INTRODUCCIÓN}

La investigación es fundamental para aumentar las bases del conocimiento científico de cualquier profesión. Para los profesionales de enfermería contribuye de forma muy importante en el desarrollo de los cuidados que se aplican a los pacientes. Según Ducharme "La investigación en enfermería explora, describe, explica y predice fenómenos que son de interés para la disciplina. Permite validar y refinar los conocimientos existentes y generar nuevos que son directa o indirectamente útiles para la práctica de la enfermería". Por tanto, podemos afirmar que las competencias en investigación son fundamentales para las enfermeras asistenciales.

Tras la Orden CIN/2134/2008, de 3 de julio, por la que se establecen los requisitos para la verificación de los títulos universitarios oficiales que habiliten para el ejercicio de la profesión de Enfermero, en España las enfermeras lo son al finalizar los estudios que condu- cen al "Grado en Enfermería" o el equivalente "Diplomado de Enfermería" (según la resolución de 30 de octubre de 2015, de la Dirección General de Política Universitaria, por la que se publica el Acuerdo del Consejo de Ministros de 30 de octubre de 2015 , por el que se determina el nivel de correspondencia al nivel del Marco Español de Cualificaciones para la Educación Superior del Título Universitario Oficial de Diplomado en Enfermería). Por tanto, nos vamos a centrar en las competencias en investigación que se le pueden suponer a una enfermera con ese nivel académico, y para ello utilizamos la clasificación propuesta por la Unidad de coordinación y desarrollo de la Investigación en Enfermería (Investén-isciii) y que las clasifica según el nivel académico de las enfermeras². Para las correspondientes al grado de enfermería, tanto genéricas como específicas, se señalan las indicadas en la Tabla 1, ordenadas según relevancia:

\section{COMPETENCIAS GENÉRICAS}

- $\quad$ Compromiso ético

- Capacidad crítica y autocrítica

- $\quad$ Capacidad para analizar y sintetizar la información

- $\quad$ Capacidad para trabajar en equipo

- Habilidad en las relaciones interpersonales

- $\quad$ Capacidad creativa

- Manejo básico del inglés

Práctica reflexiva

COMPETENCIAS ESPECÍFICAS 
- $\quad$ Conciencia de la necesidad de fundamentar la práctica enfermera en los resultados obtenidos de la investigación científica y en la mejor evidencia disponible.

- $\quad$ Capacidad para leer críticamente documentación científica bajo tutela.

- $\quad$ Capacidad para buscar y seleccionar documentación científica específica.

- Manejo básico de la realización de búsquedas en bases de datos científicas.

- $\quad$ Conocimientos básicos en metodología de la investigación.

- $\quad$ Conocimientos básicos en estadística y análisis de datos cualitativos.

- $\quad$ Conocimiento de los principios éticos establecidos para la investigación científica.

Tabla 1- Competencias de investigación correspondientes al grado de enfermería

Pero estas competencias han de ir acompañadas de ciertas habilidades y basadas en conocimientos y por eso su consecución pasa por la adquisición de habilidades específicas y conocimientos relativos al tema ${ }^{3}$. Además las competencias requieren no solo de conocimientos, sino también de comportamientos, actitudes y destrezas ${ }^{4}$.

Ya hemos descrito las competencias de investigación; en cuanto a las habilidades necesarias para investigar, entendemos que son las necesarias para llevar a cabo correctamente y con cierta facilidad los diferentes pasos del proceso investigador. Estas habilidades son muy diversas y van desde identificar una pregunta de investigación hasta defender una comunicación oral en un evento científico pasando por buscar literatura científica y analizar datos estadísticos. No siempre la adquisición de conocimientos conlleva la adquisición de habilidades, de hecho, sin experiencia es difícil ser hábiles. En el entorno docente las simulaciones proporcionan esa expe- riencia necesaria para afianzar y evaluar la adquisición de habilidades.

Pese a que la investigación es un tema de interés para las enfermeras no podemos afirmar que todos los profesionales de enfermería tengan estos conocimientos, habilidades y competencias. De hecho, una de las barreras que con mayor frecuencia identifican las enfermeras de nuestro país para la utilización de la investigación en cuidados es precisamente la falta de formación y la incapacidad para evaluar la calidad de los estudios 5 .A esto hay que añadir que incluso las enfermeras de Castilla y León piensan que no tiene suficiente apoyo por parte de las Direcciones de Enfermería y que no reciben los mismos apoyos externos que otros profesionales 6 .

Llegado a este punto parece lógico pensar que las enfermeras necesitan más formación y apoyo en investigación. En Burgos se realizó un sondeo a través del sindicato local SIScyl (encuesta de detección de necesidades) que concluyó que las enfermeras deseaban y demandaban formación continuada en investi- 
gación en cuidados, que fue el motivo que impulsó el desarrollo de esta propuesta de PCC.

Pero además debemos tener en cuenta que la motivación constituye un paso previo al aprendizaje, por lo que es necesario conocer los factores favorecedores que aumenten la motivación hacia la investigación y cuáles pueden entorpecer la misma, y utilizar esta información para adecuar las actividades formativa de manera que resulten motivadoras 7.

El objetivo de esta publicación es mostrar la estructura y contenidos de un curso de formación continuada en investigación denominado "Programa de capacitación científica" (PCC) con el que se pretende describir una propuesta que de respuesta a las necesidades detectadas y disminuir las mencionadas barreras.

\section{PLANTEAMIENTO DE LA AC- TIVIDAD DOCENTE}

Para dar respuesta a las necesidades detectadas se ha diseñado una actividad formativa denominada "Programa de capacitación científica" (PCC). El objetivo general del PCC es dotar a las enfermeras de conocimientos, habilidades y competencias para que sean capaces de producir y consumir investigación en cuidados y sigue un modelo de aprendizaje basado en el desarrollo de competencias 8 .

El PCC está compuesto por 3 módulos o cursos bien diferenciados:

- Primeros pasos para investigar: el proyecto de investigación

- Participación enfermera en eventos científicos

- Cómo publicar en una revista científica

Estos tres módulos tienen un carácter consecutivo y sus contenidos son complementarios de manera que faciliten la adquisición de los conocimientos, habilidades y competencias necesarias para producir y consumir investigación en cuidados de salud.

Los objetivos y contenidos de las 3 actividades pueden verse detallados en la tabla 2. 


\begin{tabular}{|c|c|c|}
\hline CURSO & OBJETIVOS & CONTENIDOS \\
\hline $\begin{array}{l}\text { Primeros pasos para } \\
\text { investigar: el } \\
\text { proyecto de } \\
\text { investigación }\end{array}$ & $\begin{array}{l}\text { General: } \\
\text { Capacitar a las enfermeras } \\
\text { para el diseño de un proyecto } \\
\text { de investigación en cuidados } \\
\text { Específicos: } \\
\text { 1.Identificar y plantear } \\
\text { correctamente la pregunta de } \\
\text { investigación } \\
\text { 2.Distinguir los diseños de } \\
\text { investigación y sus } \\
\text { características } \\
\text { 3.Elegir correctamente el } \\
\text { diseño de investigación que } \\
\text { responda a nuestra pregunta } \\
\text { 4.Conocer los aspectos } \\
\text { relativos a la metodología de } \\
\text { investigación escogida }\end{array}$ & $\begin{array}{l}\text { 1. ¿Qué es un proyecto de investigación? } \\
\text { 2.Planteamiento del problema a Investigar: } \\
\text { 2.1. Identificación del problema: la pregunta de } \\
\text { investigación. } \\
\text { 2.2. Justificación: Relevancia, pertinencia, } \\
\text { viabilidad y factibilidad (modelo FINEr). } \\
\text { 2.3. Hipótesis y objetivos } \\
\text { 3. Elección del diseño de investigación idóneo: } \\
\text { 3.1. Diseños cuantitativos } \\
\text { Experimentales: estudios cuasi experimentales y } \\
\text { ECAs. } \\
\text { Observacionales: Analíticos (cohortes/casos y } \\
\text { controles) y descriptivos (transversales, series } \\
\text { de casos, ecológicos). } \\
\text { 3.2. Diseños cualitativos: Fenomenología, } \\
\text { etnografía, teoría fundamentada... } \\
\text { 4.Material y método: Selección de la población } \\
\text { adecuada, tamaño de la muestra. Variables a } \\
\text { estudiar. Criterios de inclusión/exclusión. } \\
\text { Aspectos concretos del paradigma cualitativo. } \\
\text { 5. Ejecución: Aspectos prácticos sobre la } \\
\text { recogida y el análisis de la información (datos). } \\
\text { 6. Análisis de datos e interpretación de los } \\
\text { resultados } \\
\text { 7. Difusión de los resultados } \\
\text { 8. Aspectos éticos y legales } \\
\text { 9. Cronograma } \\
\text { 10. Presupuesto } \\
\text { 11. El grupo de investigación: Roles y } \\
\text { responsabilidades. }\end{array}$ \\
\hline $\begin{array}{l}\text { Participación } \\
\text { enfermera en } \\
\text { eventos científicos }\end{array}$ & 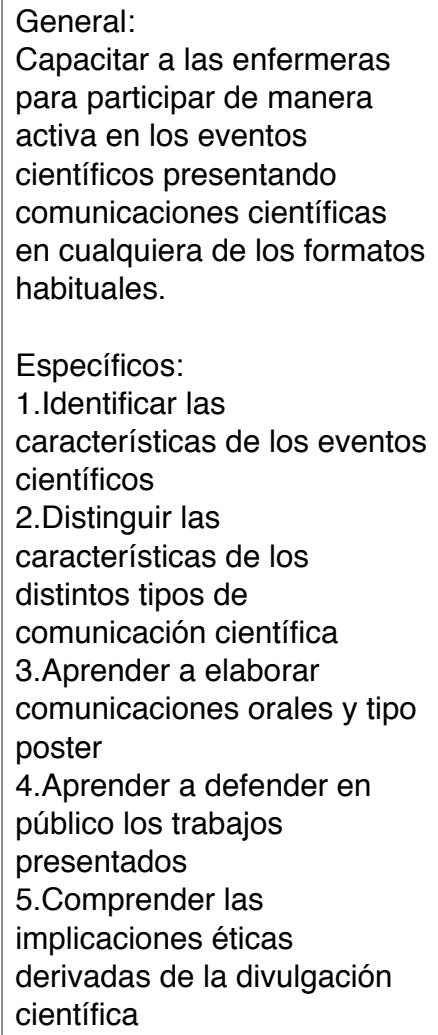 & $\begin{array}{l}\text { 1.El congreso científico: finalidad y tipos } \\
\text { 2.Modos de participación: comunicación } \\
\text { científica vs mesa de expertos } \\
\text { 3.La comunicación científica: } \\
\text { 3.1. Tipos de trabajos: trabajos de investigación, } \\
\text { casos clínicos, presentación de experiencias, } \\
\text { recensiones... } \\
\text { 3.2. Modo de presentación: ¿que presento, } \\
\text { comunicación oral o tipo poster? } \\
\text { 3.3. Cómo diseñar un poster científico: } \\
\text { Aspectos estéticos } \\
\text { Aspectos técnicos } \\
\text { Consideraciones finales } \\
\text { 3.4. Cómo elaborar una comunicación oral } \\
\text { Aspectos formales } \\
\text { Consideraciones finales } \\
\text { 3.5. Cómo defender tu trabajo científico } \\
\text { Comunicación verbal } \\
\text { Comunicación no verbal } \\
\text { Presentaciones de impacto } \\
\text { Errores comunes (y cómo evitarlos) } \\
\text { Los libros de comunicaciones } \\
\text { 4.Consideraciones finales: aspectos éticos } \\
\text { (coautoría, plagio, redundancia...) }\end{array}$ \\
\hline
\end{tabular}




\begin{tabular}{|c|c|c|}
\hline $\begin{array}{l}\text { Cómo publicar en } \\
\text { una revista científica }\end{array}$ & $\begin{array}{l}\text { General: } \\
\text { Capacitar a las enfermeras } \\
\text { para la elaboración de un } \\
\text { artículo científico. } \\
\text { Específicos: } \\
\text { 1.Conocer las características } \\
\text { de la divulgación en revistas } \\
\text { de enfermería. } \\
\text { 2.Reconocer las diferencias } \\
\text { que hay entre las revistas de } \\
\text { enfermería. } \\
\text { 3.Identificar los pasos } \\
\text { necesarios para la } \\
\text { publicación de un artículo } \\
\text { científico }\end{array}$ & $\begin{array}{l}\text { 1.Las revistas de enfermería: } \\
\text { - ¿Qué revistas existen? } \\
\text { •Funcionamiento interno: el comité editorial, } \\
\text { revisión por pares. } \\
\text { 2.Impacto y difusión de las publicaciones } \\
\text { - ¿Qué es “impacto"? Nociones básicas } \\
\text { - Índices de impacto: JCR etc. } \\
\text { 3.Elección de la revista en donde publicar. } \\
\text { Casos prácticos } \\
\text { 4.Las normas de publicación } \\
\text { - Revisión de ejemplos actuales } \\
\text { - Detalles importantes } \\
\text { - Errores a evitar } \\
\text { 5.Tipos de artículos: Carta al director, Editorial, } \\
\text { Revisión, Original, Original breve, Otros. } \\
\text { 6.La escritura científica: normas y estilo. } \\
\text { 7.Referencias, citas y bibliografía: Vancouver, } \\
\text { APA y otros } \\
\text { 8.El sistema editorial de revisión. Casos } \\
\text { prácticos } \\
\text { 9.Consideraciones ético-legales: Plagio, } \\
\text { redundancia, coautoría... } \\
\text { 10.Divulgación de tus publicaciones } \\
\text { - Ciencia 2.0. } \\
\text { - Reputación digital }\end{array}$ \\
\hline
\end{tabular}

TABLA 2- Objetivos y contenidos docentes

Cada actividad formativa tiene una duración de 10 horas distribuidas en 2 sesiones de 5 horas cada una. La inscripción en la actividad docente es global, es decir, los alumnos se inscriben en los 3 módulos.

El número máximo de participantes (alumnos) estimado es de $25 \mathrm{y}$ el control de asistencia se propone a través de un control de firmas.

\section{METODOLOGÍA DOCENTE}

Para impartir los contenidos docentes teóricos se utiliza una metodología expositiva (exposición magistral) además de la explicación de casos prácticos y ejercicios en clase. Esta metodo- logía se presenta idónea porque con ella se trata de transmitir a los estudiantes información compleja organizada desde la posición de experto con el objetivo de facilitar el aprendizaje de las condiciones y procedimientos que los receptores deben aplicar para adquirir las competencias pretendidas 9 .

El estilo de comunicación durante las exposiciones ha de ser motivacional, con mensajes de refuerzo y oferta continua de guía y asesoramiento ante la iniciativa del alumnado ${ }^{10}$.

En el cuadro 1 se exponen ejemplos de la aplicación de la metodología docente sobre los contenidos de los cursos. 
1. Exposición magistral de la teoría y debate:

- Contenido teórico: la pregunta de investigación.

Debate: ¿de dónde salen las ideas? ¿se investiga lo que realmente les interesa a las enfermeras clínicas?

2. Discusión de casos prácticos dirigida por la profesora: análisis crítico de posters científicos reales expuestos en diferentes congresos y jornadas.

3. Puesta en escena de casos simulados y corregida por la profesora: exposición de una comunicación oral de 10 minutos.

Cuadro 1- Ejemplos docentes

\section{RECURSOS DE APRENDIZA- JE Y APOYO TUTORIAL}

Las clases expositivas de desarrollan con el apoyo de medios audiovisuales. Para facilitar el seguimiento del discurso la docente utiliza esquemas en Power Point y los contenidos de la web "Enfermería Basada en el Evidencia” (www.ebevidencia.com) (Imagen 1)

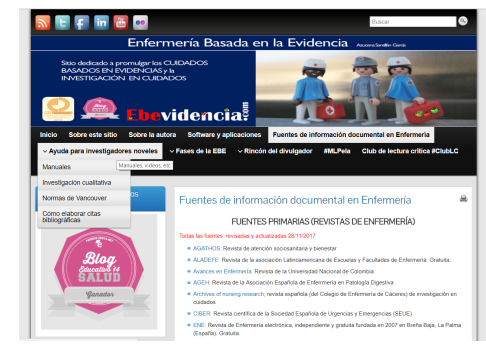

Imagen 1 - Web "Enfermería Basada en la Evidencia"
Además del blog se propone utilizar otros elementos de la web 2.0 que puedan ser útiles para la docencia y el aprendizaje de esta materia, como pueden ser las redes sociales y otras herramientas con entornos colaborativos11.

El apoyo tutorial se plantea mediante la creación de un grupo de wasap y la correspondencia online a través de email. El estilo de comunicación en las intervenciones tutoriales ha de ser motivacional y resolutivo.

\section{RESULTADOS DE APRENDI-} ZAJE Y EVALUACIÓN DE LA ACTIVIDAD

Para la evaluación de los resultados del aprendizaje se establecen diferentes mecanismos en función del área a evaluar (conocimientos, competencias o habilidades) con sus correspondientes criterios de evaluación e indicadores. Las evaluaciones se realizan de cada módulo de manera individual y las herramientas de evaluación se articulan en torno a los contendidos y finalidades de cada uno de ellos.

Evaluación de conocimientos: examen tipo test (deben responder correctamente al $70 \%$ de las preguntas).

Evaluación de competencias: expresión de comportamientos. Vinculada a esta área va la obligatoriedad de la 
PRopuesta DE UN PRograma DE CAPACitación CIENTífica
sencia física en un $\mathbf{8 0} \%$ del total de la actividad.

Evaluación de habilidades: simulaciones.

En cuanto a la evaluación de la actividad, se propone un test de satisfacción para los alumnos en el que se mida a través de una escala los siguientes ítems:

- La organización de la actividad

- El nivel de calidad de los contenidos docentes

- La calidad de las habilidades docentes del profesor

- La utilidad de los contenidos aprendidos

- La utilización de recursos audiovisuales

- La aplicación práctica de la teoría mediante los casos prácticos

- La interacción con el profesor durante la tutorización

- La duración del curso

- La valoración global del curso

Esta evaluación de la actividad se cierra con una pregunta abierta que explora las aportaciones y sugerencias de los alumnos para mejorar las próximas ediciones.

\section{CONSIDERACIONES Y RE- FLEXIONES FINALES}

El planteamiento de las actividades formativas orientadas a la consecución de competencias pretende dar un paso más en la formación sobre investigación. Actualmente la oferta formativa en investigación es amplia, pero es necesario facilitar no solo conocimientos teóricos sino práctica, tutorización y motivación.

Orientando los contenidos de las actividades formativas hacia la consecución de las competencias adecuadas al nivel académico de las enfermeras, se intenta garantizar la adecuación y pertinencia de estas y por ende su éxito en resultados de aprendizaje y calidad de la actividad.

Este PCC se implantó en 2016 y ha sido evaluado analizando los resultados de la propia formación (resultados del aprendizaje y evaluación de la actividad) por un lado, y el impacto sobre la actitud y la motivación de las participantes por otro. El PCC obtuvo buenos resultados de aprendizaje y también incidió positivamente en la motivación de las alumnas, especialmente en las áreas "conocimientos y preparación", "recursos disponibles y apoyos" y "motivaciones".12

Además, con el objetivo de analizar la percepción de las enfermeras en 
cuanto a la utilidad de la investigación y las facilidades/dificultades que encuentran para investigar, durante las clases de la segunda edición de este PCC (realizada entre 2016 y 2017) se realizó una exploración cualitativa a través de la observación participante en el aula. Los resultados de esta exploración conducen a la conclusión de que las enfermeras quieren investigar, pero sienten que es una labor ingrata porque no tienen apoyos y porque posteriormente no se aplican los resultados en la práctica diaria ${ }^{13}$. Esto coincide con lo detectado por Cepeda en Castilla y León hace una decada ${ }^{6}$ y nos hace cuestionarnos si las políticas y estrategias del fomento de la investigación en cuidados en esta región han sido suficientes. 


\section{BIBLIOGRAFÍA}

1. Ducharme F. La recherche en sciences infirmiers, des défs à relever. Can J Nurs Res. 1997; 29(4): 123- 40.

2. Fuentelsaz-Gallego, Carmen, Susana NavalpotroPascual, and María Ruzafa-Martínez. Competencias en investigación: propuesta de la Unidad de coordinación y desarrollo de la Investigación en Enfermería (Investén-isciii). Enfermería clínica 17.3 (2007): 117-127.

3. Gilar-Corbi, R. (2003). Adquisición de habilidades cognitivas. Factores en el desarrollo inicial de la competencia experta (Doctoral dissertation, Universitat d'Alacant-Universidad de Alicante). [Fecha de consulta: 31 de enero de 2019] Disponible en:< http://hdl.handle.net/10045/9906 > ISBN 84-688-5125-6

4. Sánchez-Cabaco, A. (2005). Competencias y habilidades de la nueva reforma en educación superior. Papeles Salmantinos De Educación, (5). [Fecha de consulta: 22 de enero de 2020] Recuperado de ht t p://summa.upsa.es/high.raw? id $=0000029491 \&$ name $=00000001$. original.pdf\&atta chment=0000029491.pdf

5. Moreno-Casbas, Teresa, et al. Barreras para la utilización de la investigación. Estudio descriptivo en profesionales de enfermería de la práctica clínica y en investigadores activos. Enfermería clínica 20.3 (2010): 153-164

6. Cepeda Diez JM, San Román Calvo MJ, Vaca Espinel, V, Álvarez Vilas C, Millán Cuesta B. Actitud y motivación de la Enfermería de Castilla y León hacia la investigación. Revista Enfermería Castilla y León 2010 2(2):10-28 [Fecha de consulta: 15 de marzo de 2019] Disponible en:< http:// www.revistaenfermeriacyl.com/index.php/revistaenfermeriacyl/article/view/48> ISSN 1989-3885
7. Mollon, D., Fields, W., Gallo, A. M., Wagener, R., Soucy, J., Gustafson, B., \& Kim, S. C. (2012). Staff practice, attitudes, and knowledge/skills regarding evidence-based practice before and after an educational intervention. Journal of Continuing Education in Nursing, 43(9), 411-419. doi: 10.3928/00220124-20120716-89

8. De Miguel, M. (coord.) (2005). Modalidades de enseñanza centradas en el desarrollo de competencias. Orientaciones para promover el cambio metodológico en el EEES. Oviedo: Ediciones de la Universidad de Oviedo

9. Tronchoni, H., Izquierdo, C., y Anguera, M. T. (2018). Interacción participativa en las clases magistrales: fundamentación y construcción de un instrumento de observación. Publicaciones, 48(1), 77-95.doi:10.30827/publicaciones.v48i1.7331

10. Huertas, Juan Antonio; Ardura, Aranzazu; Nieto, Carmen, Cómo estudiar el papel que el desempeño docente y las formas de comunicación juegan en el clima motivacional del aula. Sugerencias para un trabajo empírico. Educação [en linea] 2008, 31 (Enero-Abril) : [Fecha de consulta: 23 de enero de 2020] Disponible en:<http://148.215.2.11/ articulo.oa?id=84806402> ISSN 0101-465X

11.Gómez Sánchez, A., \& Escudero Gómez, C. (2017). Recursos 2.0 indispensables para profesionales en ciencias de la salud. Metas De Enfermería, 20(2), 66-71. doi: 10.35667/metasenf. 2019.20.1003081039

12.Santillan-Garcia, A. (2019). Diseño, aplicación y evaluación de un modelo de docencia presencial con internet en enfermería (Doctorado). Universidad de Burgos.

13.Santillán-García, A.; Estébanez Lucio, N (2017) .Cultura de investigación: Percepción de las enfermeras. Libro de actas del I Congreso Internacional de Intervención e Investigación en Salud. Vol I, 2583-2583. Disponible en https://formacionasunivep.com/ciiis/get/acta_individual/AI777 